\title{
LONG-TIME-STEP INTEGRATORS FOR ALMOST-ADIABATIC QUANTUM DYNAMICS*
}

\author{
TOBIAS JAHNKE ${ }^{\dagger}$
}

\begin{abstract}
The highly oscillatory solution of a singularly perturbed Schrödinger equation with time-dependent Hamiltonian is computed numerically. The new time-symmetric integrators presented here can be used efficiently with step sizes significantly larger than those required by traditional schemes. This is achieved by a transformation of the problem and an expansion technique for integrals over the oscillating components. The error behavior in the adiabatic case is thoroughly analyzed, and the performance of the methods is illustrated both in an almost-adiabatic setup and in an avoided energy level crossing, where nonadiabatic state transitions occur.
\end{abstract}

Key words. singularly perturbed Schrödinger equation, highly oscillatory problem, numerical integrator, multiple time scales

AMS subject classifications. 65L05, 65L70, 65M15, 65M20, 81-04, 81-08

DOI. $10.1137 / \mathrm{S} 1064827502411316$

1. Introduction. Singularly perturbed Schrödinger equations of the form

$$
i \dot{\psi}(t)=\frac{1}{\varepsilon} H(t) \psi(t)
$$

with a small parameter $0<\varepsilon \ll 1$ and a time-dependent Hamiltonian $H(t)$ represented by a finite-dimensional matrix have been studied since the early days of quantum mechanics, yielding considerable insight into the nature of quantum systems $[2,8,22]$. Furthermore, these and similar equations often play an important role when mixed quantum-classical models are used to include quantum effects into simulations of molecular dynamics (see, e.g., papers and references in $[5,18]$ ).

Two of these models are the surface hopping approach $[6,7,21]$ and the system known as Ehrenfest, mean field, or QCMD (quantum-classical molecular dynamics) $[3,4,6,18,20]$. In both models, the nuclei of the molecule are considered as classical particles moving according to a Newtonian equation of motion, but the force depends on the behavior of the electrons which remain quantum particles and are described by the Schrödinger equation (1.1). The parameter $\varepsilon$ is typically defined by the square root of the mass ratio $m_{q} / m_{c}$. If $m_{q}$, the mass of the quantum particles, is significantly less than $m_{c}$, the mass of the classical particles, then the constant $\varepsilon=\sqrt{m_{q} / m_{c}}$ takes a small value, e.g., $\varepsilon \approx 10^{-2}$ for electron-ion interactions. As a consequence, the wave function $\psi$ oscillates on a fast time scale $\sim \varepsilon$, whereas the classical degrees of freedom move on a comparatively slow time scale $\sim 1$. Hence, solving the Schrödinger equation (1.1) is the numerically critical part in both models.

If any traditional solver such as, e.g., a Runge-Kutta method, is chosen to approximate the quantum part, the time step size $h$ has to be much smaller than the frequency of the oscillations, i.e., $h \ll \varepsilon$. This necessitates a huge number of time

${ }^{*}$ Received by the editors July 15, 2002; accepted for publication (in revised form) October 21, 2003; published electronically July 23, 2004. This work was done at the University of Tübingen and supported by the DFG Priority Program 1095 Analysis, Modeling and Simulation of Multiscale Problems.

http://www.siam.org/journals/sisc/25-6/41131.html

${ }^{\dagger}$ Freie Universität Berlin, Institut für Mathematik II, BioComputing Group, Arnimallee 2-6, D-14195 Berlin, Germany (Tobias.Jahnke@math.fu-berlin.de). 
steps and consequently a huge number of evaluations of the time-dependent Hamiltonian $H(t)$. In real-life applications, however, evaluating $H(t)$ is a computationally expensive procedure because the matrix $H(t)$ has to be derived from the Hamiltonian of the full molecular Schrödinger equation by an intricate modeling step. Thus, the aim of this paper is to devise and analyze two new integrators which

- can be used with a big step size $h>\varepsilon$ to compute the oscillating solution of (1.1), and

- need only one evaluation and (partial) diagonalization of $H(t)$ per time step. The numerical methods proposed so far for $(1.1)[1,9,10,12,13,14,19,20]$ are based on the premise of a fixed positive $\varepsilon$. They require either many evaluations of $H(t)$ and/or a small $h \ll \varepsilon$. For $\varepsilon \rightarrow 0$ and $h>\varepsilon$, neither of these methods approximates the adiabatic limit equation, which is given by the Born-Fock quantum-adiabatic theorem [2].

In [16], several numerical methods were designed for the above demands. However, these methods are sensitive to resonances for certain choices of the step size $h$. By construction, an accuracy of $\mathcal{O}\left(h^{2}\right)$ is obtained only if certain oscillatory $\mathcal{O}\left(h^{2}\right)$ terms included in the local error do not sum up. Our aim is to avoid this undesirable effect with improved integrators having a local error of $\mathcal{O}\left(h^{3}\right)$ and hence providing an accuracy of $\mathcal{O}\left(h^{2}\right)$ independently of any resonances.

The paper is organized as follows. In section 2, the problem (1.1) will be transformed by means of the eigendecomposition. This allows a change from the highly oscillatory wave function $\psi$ to a smoother variable $\eta$. A first time-reversible method for the transformed equation is presented in section 3. Some fundamental ideas for the construction were developed in [16], but the approach is refined by the inclusion of higher order terms and an expansion technique for the oscillatory variables, which appear as integrals over exponential functions. Section 4 gives a short summary of the concept of Magnus methods. It will turn out that, similarly to the first scheme, a Magnus-type integrator can be derived. Numerical examples are shown in section 5. The methods are applied both to an almost-adiabatic situation and an avoided energy level crossing with nonadiabatic state transitions. A comparison with several traditional schemes demonstrates that the accuracy of the new integrators is far better and that they generate very precise results even when large step sizes $h>\varepsilon$ are used. These observations are confirmed by a detailed error analysis in section 6 .

2. Reformulation of the problem. The numerical methods will make use of the observation that, instead of computing $\psi$ from the Schrödinger equation (1.1) directly, it is more favorable to transform the problem as it was proposed in [16]. For convenience of the reader, we repeat the procedure here.

2.1. Assumptions. It will be assumed that, for all $t \in\left[t_{0}, t_{\text {end }}\right], H(t)$ is a real symmetric matrix, and that the entries of $H(t)$ change slowly compared to the oscillations of $\psi$. Then $H(t)$ can be diagonalized as

$$
H(t)=Q(t) \Lambda(t) Q(t)^{T}, \quad \Lambda(t)=\operatorname{diag}\left(\lambda_{k}(t)\right)
$$

with an orthogonal matrix $Q(t)$ and a real diagonal matrix $\Lambda(t)$ containing the eigenvalues $\lambda_{k}(t)$ of $H(t)$. The decomposition is sufficiently smooth, i.e., both $t \longmapsto Q(t)$ and $t \longmapsto \Lambda(t)$ are three times continuously differentiable, and the first to third derivatives are bounded independently of $\varepsilon$. Moreover, it is assumed that the eigenvalues as functions in time do not intersect and remain separated; i.e., there is a $\delta_{\min }>0$ 
such that for any pair $\lambda_{k}(t)$ and $\lambda_{l}(t)$ with $k \neq l$, the lower bound

$$
\left|\lambda_{k}(t)-\lambda_{l}(t)\right| \geq \delta_{\min }
$$

holds for all $t \in\left[t_{0}, t_{\text {end }}\right]$.

If the dimension of $H(t)$ is so large that a complete diagonalization cannot be carried out, only the subspace spanned by the eigenvectors corresponding to a sufficiently large number of lower eigenvalues may be considered. Such a reduction of the dimension is motivated by the version of the quantum-adiabatic theorem presented in [16]. In the surface hopping approach, however, the matrix $H(t)$ typically is of small size (but nevertheless expensive to evaluate), because $H(t)$ has to be diagonalized anyway due to the physical model: The coefficients of the wave function with respect to the eigenbasis have to be computed in order to determine the probability of state transitions $[6,7,21]$. Thus, the diagonalization is already available for the numerical integration of the corresponding Schrödinger equation.

2.2. Changing variables. A new function $\eta$ can be defined by the relation

$$
Q(t)^{T} \psi(t)=\exp \left(-\frac{i}{\varepsilon} \Phi(t)\right) \eta(t)
$$

where $\Phi(t)$ is the diagonal matrix containing the integrals over the eigenvalues:

$$
\Phi(t)=\int_{t_{0}}^{t} \Lambda(s) d s, \quad \Phi=\operatorname{diag}\left(\phi_{j}\right)
$$

Up to an oscillating phase, $\eta$ is the coefficient vector of $\psi$ with respect to the eigenbasis. Differentiating (2.2) and using (1.1) shows that $\eta$ solves the differential equation

$$
\dot{\eta}(t)=\exp \left(\frac{i}{\varepsilon} \Phi(t)\right) W(t) \exp \left(-\frac{i}{\varepsilon} \Phi(t)\right) \eta(t)
$$

with two rapidly oscillating diagonal matrices $\exp ( \pm i \Phi(t) / \varepsilon)$ and a real coupling matrix $W=\dot{Q}^{T} Q$. It will be used frequently that $W$ is skew-symmetric and has zero diagonal entries.

The above transformation was already used in [21], but it was noticed only in [16] that it enables the construction of very efficient numerical integrators. From the point of view of numerical analysis, the motivation for transforming the problem is the fact that the right-hand side of (2.3) stays bounded independently from $\varepsilon$. Hence, the new function $\eta$ is still highly oscillatory but smoother than $\psi$. Another good reason for working with $\eta$ rather than $\psi$ is provided by the quantum-adiabatic theorem of Born and Fock [2], stating that under the separation condition (2.1), the absolute values of the coefficients in the eigenbasis representation of the wave function $\psi$ are adiabatic invariants of the motion as $\varepsilon \rightarrow 0$. In fact, a simple integration by parts proves [16] that under condition (2.1)

$$
\eta(t)=\eta\left(t_{0}\right)+\mathcal{O}(\varepsilon)
$$

holds uniformly on bounded time intervals as $\varepsilon \rightarrow 0$. 
2.3. Notation. It will be helpful to use the following notation. $E(\Phi)$ stands for a matrix with entries $e_{k l}(\Phi)$ defined by

$$
e_{k l}(\Phi)=\left\{\begin{array}{cl}
\exp \left(\frac{i}{\varepsilon}\left(\phi_{k}-\phi_{l}\right)\right) & \text { if } k \neq l \\
0 & \text { else. }
\end{array}\right.
$$

Denoting the entrywise product of matrices by $\bullet$, equation (2.3) can be restated as

$$
\dot{\eta}=(E(\Phi) \bullet W) \eta
$$

Moreover, $D(\Lambda)$ and $D^{-}(\Lambda)$ are defined as the matrices with entries

$$
d_{k l}(\Lambda)=\lambda_{k}-\lambda_{l}, \quad d_{k l}^{-}(\Lambda)=\left\{\begin{array}{cl}
\left(\lambda_{k}-\lambda_{l}\right)^{-1} & \text { if } k \neq l, \\
0 & \text { else. }
\end{array}\right.
$$

The same definitions apply if $\Phi$ or $\Lambda$ are replaced by any other diagonal matrix. Note that $D^{-}(\Lambda)$ is not the inverse of $D(\Lambda)$, but the entrywise product yields $D(\Lambda) \bullet D^{-}(\Lambda) \bullet$ $W=W$ for every matrix $W$ with zero diagonal.

3. Method I. After these preparations, a time-symmetric method for the new equation (2.5) can be derived. The approach is related to that of [16], but the concept is extended in order to obtain a more accurate integrator requiring similar computational work.

3.1. The basic equations. Let $h$ be the step size and fix a time $t_{n}=t_{0}+n h$. Integrating (2.5) from $t_{n-1}$ to $t_{n+1}$ and from $t_{n}$ to $t_{n}+\theta h$ yields

$$
\begin{aligned}
& \eta\left(t_{n+1}\right)=\eta\left(t_{n-1}\right)+h \int_{-1}^{1}\left(E\left(\Phi\left(t_{n}+\theta h\right)\right) \bullet W\left(t_{n}+\theta h\right)\right) \eta\left(t_{n}+\theta h\right) d \theta, \\
& \eta\left(t_{n}+\theta h\right)=\eta\left(t_{n}\right)+h \int_{0}^{\theta}\left(E\left(\Phi\left(t_{n}+\sigma h\right)\right) \bullet W\left(t_{n}+\sigma h\right)\right) \eta\left(t_{n}+\sigma h\right) d \sigma
\end{aligned}
$$

respectively. In (3.1), the expression $\eta\left(t_{n}+\theta h\right)$ is substituted by the right-hand side of (3.2), and the intermediate value $W\left(t_{n}+\theta h\right)$ is replaced by the Taylor expansion

$$
W\left(t_{n}+\theta h\right)=W\left(t_{n}\right)+\theta h \dot{W}\left(t_{n}\right)+\mathcal{O}\left(\theta^{2} h^{2}\right) .
$$

Using $\eta\left(t_{n}+\sigma h\right)=\eta\left(t_{n}\right)+\mathcal{O}(\sigma h)$, this leads to

$$
\eta\left(t_{n+1}\right)=\eta\left(t_{n-1}\right)+h \alpha_{n} \eta\left(t_{n}\right)+h^{2} \beta_{n} \eta\left(t_{n}\right)+h^{2} \gamma_{n} \eta\left(t_{n}\right)+\mathcal{O}\left(h^{3}\right)
$$

with

$$
\begin{aligned}
\alpha_{n} & =\int_{-1}^{1} E\left(\Phi\left(t_{n}+\theta h\right)\right) d \theta \bullet W\left(t_{n}\right), \\
\beta_{n} & =\int_{-1}^{1} \theta E\left(\Phi\left(t_{n}+\theta h\right)\right) d \theta \bullet \dot{W}\left(t_{n}\right), \\
\gamma_{n} & =\int_{-1}^{1}\left(E\left(\Phi\left(t_{n}+\theta h\right)\right) \bullet W\left(t_{n}\right)\right) \int_{0}^{\theta}\left(E\left(\Phi\left(t_{n}+\sigma h\right)\right) \bullet W\left(t_{n}\right)\right) d \sigma d \theta .
\end{aligned}
$$


These equations are the starting point for the construction of the numerical method. The task for the remainder of this section is to approximate $\alpha_{n}, \beta_{n}$, and $\gamma_{n}$. First, $W\left(t_{n}\right)$ and $\dot{W}\left(t_{n}\right)$ are replaced by the symmetric difference quotients

$$
\begin{aligned}
W\left(t_{n}\right) \approx W_{n} & =\frac{1}{2 h}\left(Q\left(t_{n+1}\right)-Q\left(t_{n-1}\right)\right)^{T} Q\left(t_{n}\right), \\
\dot{W}\left(t_{n}\right) \approx \dot{W}_{n}= & \frac{1}{h}\left(W_{n+\frac{1}{2}}-W_{n-\frac{1}{2}}\right), \\
W_{n+\frac{1}{2}} & =\frac{1}{2 h}\left(Q\left(t_{n+1}\right)-Q\left(t_{n}\right)\right)^{T}\left(Q\left(t_{n+1}\right)+Q\left(t_{n}\right)\right) .
\end{aligned}
$$

Next, we insert the Taylor expansion

$$
\Phi\left(t_{n}+\theta h\right) \approx \Phi\left(t_{n}\right)+\theta h \Lambda\left(t_{n}\right)+\frac{1}{2} \theta^{2} h^{2} \dot{\Lambda}\left(t_{n}\right)
$$

into the argument of $E(\cdot)$ in $\alpha_{n}, \beta_{n}$, and $\gamma_{n} . \dot{\Lambda}\left(t_{n}\right)$ is approximated via

$$
\dot{\Lambda}\left(t_{n}\right) \approx \dot{\Lambda}_{n}=\frac{1}{2 h}\left(\Lambda\left(t_{n+1}\right)-\Lambda\left(t_{n-1}\right)\right)
$$

whereas the integral $\Phi\left(t_{n}\right)=\int_{t_{0}}^{t_{n}} \Lambda(s) d s$ is calculated by the Simpson rule

$$
\Phi\left(t_{n}\right) \approx \Phi_{n}=\Phi_{n-2}+\frac{h}{3}\left(\Lambda\left(t_{n}\right)+4 \Lambda\left(t_{n-1}\right)+\Lambda\left(t_{n-2}\right)\right)
$$

3.2. Computing the integrals. After these substitutions, the matrices (3.4), (3.5), and (3.6) have turned into

$$
\begin{aligned}
\widetilde{\alpha}_{n}=E\left(\Phi_{n}\right) \bullet \int_{-1}^{1} E\left(\theta h \Lambda\left(t_{n}\right)+\frac{1}{2} \theta^{2} h^{2} \dot{\Lambda}_{n}\right) d \theta \bullet W_{n} \\
\widetilde{\beta}_{n}=E\left(\Phi_{n}\right) \bullet \int_{-1}^{1} \theta E\left(\theta h \Lambda\left(t_{n}\right)+\frac{1}{2} \theta^{2} h^{2} \dot{\Lambda}_{n}\right) d \theta \bullet \dot{W}_{n} \\
\widetilde{\gamma}_{n}=\left(E\left(\Phi_{n}\right) \bullet \int_{-1}^{1} E\left(\theta h \Lambda\left(t_{n}\right)+\frac{1}{2} \theta^{2} h^{2} \dot{\Lambda}_{n}\right) \bullet W_{n}\right) \\
\times\left(E\left(\Phi_{n}\right) \bullet \int_{0}^{\theta} E\left(\sigma h \Lambda\left(t_{n}\right)+\frac{1}{2} \sigma^{2} h^{2} \dot{\Lambda}_{n}\right) \bullet W_{n}\right) d \sigma d \theta
\end{aligned}
$$

(To disambiguate notation, the symbol $\times$ will be used where necessary to signify matrix multiplication.) Now the oscillating exponentials have to be integrated in an efficient way. In [16], this was done by simply calculating the integrals analytically, but unfortunately this is impossible now — at least for the double integral in $\widetilde{\gamma}_{n}$-due to the second order term in the Taylor expansion of $\Phi$, which was omitted in [16]. A feasible way out consists in expanding the integrals up to a sufficiently high order of 
$h$. First, consider the integral in $\widetilde{\beta}_{n}$. Integrating by parts yields ${ }^{1}$

$$
\begin{aligned}
\int_{-1}^{1} \theta E\left(\theta h \Lambda\left(t_{n}\right)\right) & \bullet E\left(\frac{1}{2} \theta^{2} h^{2} \dot{\Lambda}_{n}\right) d \theta \\
=E\left(\theta h \Lambda\left(t_{n}\right)\right. & \left.+\frac{1}{2} \theta^{2} h^{2} \dot{\Lambda}_{n}\right) \\
& \left.\bullet\left(\theta \frac{\varepsilon}{i h} D^{-}\left(\Lambda\left(t_{n}\right)\right)-\left(\frac{\varepsilon}{i h} D^{-}\left(\Lambda\left(t_{n}\right)\right)\right)^{\bullet 2}\right)\right|_{\theta=-1} ^{1} \\
-\int_{-1}^{1} E\left(\theta h \Lambda\left(t_{n}\right)+\frac{1}{2} \theta^{2} h^{2} \dot{\Lambda}_{n}\right) & \bullet\left(\theta \frac{\varepsilon}{i h} D^{-}\left(\Lambda\left(t_{n}\right)\right)-\left(\frac{\varepsilon}{i h} D^{-}\left(\Lambda\left(t_{n}\right)\right)\right)^{\bullet 2}\right) \bullet \theta \frac{i h^{2}}{\varepsilon} D\left(\dot{\Lambda}_{n}\right) d \theta .
\end{aligned}
$$

Since the last integral is of $\mathcal{O}(h)$, this gives

$$
\begin{aligned}
& \int_{-1}^{1} \theta E\left(\theta h \Lambda\left(t_{n}\right)+\frac{1}{2} \theta^{2} h^{2} \dot{\Lambda}_{n}\right) d \theta=\mathcal{T}_{3}\left(t_{n}\right)+\mathcal{O}(h) \\
& \mathcal{T}_{3}\left(t_{n}\right)=\frac{\varepsilon}{i h} D^{-}\left(\Lambda\left(t_{n}\right)\right) \bullet \mathcal{T}_{2}\left(t_{n}\right)-\left(\frac{\varepsilon}{i h} D^{-}\left(\Lambda\left(t_{n}\right)\right)\right)^{\bullet 2} \bullet \mathcal{T}_{1}\left(t_{n}\right) \\
& \mathcal{T}_{2}\left(t_{n}\right)=\left.\theta \cdot E\left(\theta h \Lambda\left(t_{n}\right)+\frac{1}{2} \theta^{2} h^{2} \dot{\Lambda}_{n}\right)\right|_{\theta=-1} ^{1} \\
& \mathcal{T}_{1}\left(t_{n}\right)=\left.E\left(\theta h \Lambda\left(t_{n}\right)+\frac{1}{2} \theta^{2} h^{2} \dot{\Lambda}_{n}\right)\right|_{\theta=-1} ^{1}
\end{aligned}
$$

The integral in $\widetilde{\alpha}_{n}$ is treated in a similar way. Integrating by parts and using (3.17) turns it into

$$
\begin{aligned}
& \int_{-1}^{1} E\left(\theta h \Lambda\left(t_{n}\right)\right) \bullet E\left(\frac{1}{2} \theta^{2} h^{2} \dot{\Lambda}_{n}\right) d \theta \\
& =\frac{\varepsilon}{i h} D^{-}\left(\Lambda\left(t_{n}\right)\right) \bullet \mathcal{T}_{1}\left(t_{n}\right) \\
& -\frac{\varepsilon}{i h} D^{-}\left(\Lambda\left(t_{n}\right)\right) \bullet \int_{-1}^{1} E\left(\theta h \Lambda\left(t_{n}\right)+\frac{1}{2} \theta^{2} h^{2} \dot{\Lambda}_{n}\right) \bullet \theta \frac{i h^{2}}{\varepsilon} D\left(\dot{\Lambda}_{n}\right) d \theta \\
& =\frac{\varepsilon}{i h} D^{-}\left(\Lambda\left(t_{n}\right)\right) \bullet \mathcal{T}_{1}\left(t_{n}\right) \\
& -h D^{-}\left(\Lambda\left(t_{n}\right)\right) \bullet D\left(\dot{\Lambda}_{n}\right) \bullet \mathcal{T}_{3}\left(t_{n}\right)+\mathcal{O}\left(h^{2}\right) .
\end{aligned}
$$

The term $\widetilde{\gamma}_{n}$ is slightly more complicated due to the double integral and the presence of both matrix multiplication and entrywise multiplication. By definition,

$$
\left(E(\Phi) \bullet M_{1}\right)\left(E(\Phi) \bullet M_{2}\right)=(E(\Phi)+I) \bullet\left(M_{1} M_{2}\right)
$$

holds for all diagonal matrices $\Phi$ and arbitrary $M_{1}, M_{2} \in \mathbb{R}^{N \times N}$ with zero diagonal,

\footnotetext{
${ }^{1}$ Here, $X^{\bullet 2}$ denotes the entrywise power, i.e., $X^{\bullet 2}=X \bullet X$.
} 
where $I$ is the identity. Integration by parts, similar to that above, leads to

$$
\begin{aligned}
\widetilde{\gamma}_{n}= & \left(E\left(\Phi_{n}\right) \bullet \int_{-1}^{1} E\left(\theta h \Lambda\left(t_{n}\right)+\frac{1}{2} \theta^{2} h^{2} \dot{\Lambda}_{n}\right) \bullet W_{n}\right) \\
& \times\left(\frac{\varepsilon}{i h} D^{-}\left(\Lambda\left(t_{n}\right)\right) \bullet\left(E\left(\Phi_{n}+\theta h \Lambda\left(t_{n}\right)+\frac{1}{2} \theta^{2} h^{2} \dot{\Lambda}_{n}\right)-E\left(\Phi_{n}\right)\right) \bullet W_{n}\right) d \theta+\mathcal{O}(h),
\end{aligned}
$$

and with (3.22) and (3.23) we obtain

$$
\begin{aligned}
& \mathcal{C}_{n}= \mathcal{T}_{4}\left(t_{n}\right) \bullet\left(W_{n} \mathcal{T}_{5}\left(t_{n}\right)\right) \\
& \quad-\left(E\left(\Phi_{n}\right) \bullet \mathcal{T}_{1}\left(t_{n}\right) \bullet \mathcal{T}_{5}\left(t_{n}\right)\right)\left(E\left(\Phi_{n}\right) \bullet \mathcal{T}_{5}\left(t_{n}\right)\right), \\
& \mathcal{T}_{4}\left(t_{n}\right)=E\left(\Phi_{n}\right) \bullet \frac{\varepsilon}{i h} D^{-}\left(\Lambda\left(t_{n}\right)\right) \bullet \mathcal{T}_{1}\left(t_{n}\right)+2 I, \\
& \mathcal{T}_{5}\left(t_{n}\right)=\frac{\varepsilon}{i h} D^{-}\left(\Lambda\left(t_{n}\right)\right) \bullet W_{n}
\end{aligned}
$$

as an approximation to $\widetilde{\gamma}_{n}$ of $\mathcal{O}(h)$. Summarizing, Method I reads as follows.

\subsection{The algorithm of Method I.}

1. Let $\eta_{n}, \eta_{n-1}, Q\left(t_{n}\right), Q\left(t_{n-1}\right), \Lambda\left(t_{n}\right), \Lambda\left(t_{n-1}\right), \Phi_{n}, \Phi_{n-1}, W_{n-\frac{1}{2}}$ be given from the previous steps.

2. Evaluate $H\left(t_{n+1}\right)$ and diagonalize:

$$
H\left(t_{n+1}\right)=Q\left(t_{n+1}\right) \Lambda\left(t_{n+1}\right) Q\left(t_{n+1}\right)^{T} .
$$

3. Compute $W_{n}$ and its derivative:

$$
\begin{aligned}
W_{n} & =\frac{1}{2 h}\left(Q\left(t_{n+1}\right)-Q\left(t_{n-1}\right)\right)^{T} Q\left(t_{n}\right), \\
W_{n+\frac{1}{2}} & =\frac{1}{2 h}\left(Q\left(t_{n+1}\right)-Q\left(t_{n}\right)\right)^{T}\left(Q\left(t_{n+1}\right)+Q\left(t_{n}\right)\right), \\
\dot{W}_{n} & =\frac{1}{h}\left(W_{n+\frac{1}{2}}-W_{n-\frac{1}{2}}\right) .
\end{aligned}
$$

4. Compute $\dot{\Lambda}_{n}=\frac{1}{2 h}\left(\Lambda\left(t_{n+1}\right)-\Lambda\left(t_{n-1}\right)\right)$.

5. Expand the integrals:

$$
\begin{aligned}
& \mathcal{T}_{1}\left(t_{n}\right)=E\left(h \Lambda\left(t_{n}\right)+\frac{1}{2} h^{2} \dot{\Lambda}_{n}\right)-E\left(-h \Lambda\left(t_{n}\right)+\frac{1}{2} h^{2} \dot{\Lambda}_{n}\right), \\
& \mathcal{T}_{2}\left(t_{n}\right)=E\left(h \Lambda\left(t_{n}\right)+\frac{1}{2} h^{2} \dot{\Lambda}_{n}\right)+E\left(-h \Lambda\left(t_{n}\right)+\frac{1}{2} h^{2} \dot{\Lambda}_{n}\right), \\
& \mathcal{T}_{3}\left(t_{n}\right)=\frac{\varepsilon}{i h} D^{-}\left(\Lambda\left(t_{n}\right)\right) \bullet \mathcal{T}_{2}\left(t_{n}\right)-\left(\frac{\varepsilon}{i h} D^{-}\left(\Lambda\left(t_{n}\right)\right)\right)^{\bullet 2} \bullet \mathcal{T}_{1}\left(t_{n}\right), \\
& \mathcal{T}_{4}\left(t_{n}\right)=E\left(\Phi_{n}\right) \bullet \frac{\varepsilon}{i h} D^{-}\left(\Lambda\left(t_{n}\right)\right) \bullet \mathcal{T}_{1}\left(t_{n}\right)+2 I, \\
& \mathcal{T}_{5}\left(t_{n}\right)=\frac{\varepsilon}{i h} D^{-}\left(\Lambda\left(t_{n}\right)\right) \bullet W_{n} .
\end{aligned}
$$


6. Compute

$$
\begin{aligned}
\mathcal{A}_{n}=E\left(\Phi_{n}\right) \bullet\left(\frac{\varepsilon}{i h} D^{-}\left(\Lambda\left(t_{n}\right)\right) \bullet \mathcal{T}_{1}\left(t_{n}\right)\right. & \left.\quad-h D^{-}\left(\Lambda\left(t_{n}\right)\right) \bullet D\left(\dot{\Lambda}_{n}\right) \bullet \mathcal{T}_{3}\left(t_{n}\right)\right) \bullet W_{n}, \\
& \mathcal{B}_{n}=E\left(\Phi_{n}\right) \bullet \mathcal{T}_{3}\left(t_{n}\right) \bullet \dot{W}_{n}, \\
\mathcal{C}_{n}= & \mathcal{T}_{4}\left(t_{n}\right) \bullet\left(W_{n} \mathcal{T}_{5}\left(t_{n}\right)\right)-\left(E\left(\Phi_{n}\right) \bullet \mathcal{T}_{1}\left(t_{n}\right) \bullet \mathcal{T}_{5}\left(t_{n}\right)\right)\left(E\left(\Phi_{n}\right) \bullet \mathcal{T}_{5}\left(t_{n}\right)\right) .
\end{aligned}
$$

7. Update the quantum vector:

$$
\eta_{n+1}=\eta_{n-1}+h \mathcal{A}_{n} \eta_{n}+h^{2} \mathcal{B}_{n} \eta_{n}+h^{2} \mathcal{C}_{n} \eta_{n}
$$

8. Compute $\Phi_{n+1}$ by the Simpson rule:

$$
\Phi_{n+1}=\Phi_{n-1}+\frac{h}{3}\left(\Lambda\left(t_{n+1}\right)+4 \Lambda\left(t_{n}\right)+\Lambda\left(t_{n-1}\right)\right) .
$$

9. Update the wave function:

$$
\psi_{n+1}=Q\left(t_{n+1}\right) \exp \left(-\frac{i}{\varepsilon} \Phi_{n+1}\right) \eta_{n+1} .
$$

Postprocessing step: Normalize all $\eta_{n}$ and $\psi_{n}$. This may be advantageous because the algorithm is not norm-preserving, although the Schrödinger equation (1.1) and its unitary transformation (2.5) are known to have a unitary propagator.

Remark 1. Care should be taken that the ordering of the eigenvalues in $\Lambda$ remains the same in all time steps, and that the diagonalization of $H(t)$ does not produce artificial sign changes of the eigenvectors (the columns of $Q$ ).

Remark 2. Although $W\left(t_{n}\right)$ and $\dot{W}\left(t_{n}\right)$ are skew-symmetric matrices, the corresponding approximations $W_{n}$ and $\dot{W}_{n}$ are only "nearly skew-symmetric." In particular, they may include small nonzero diagonal entries, which can arise from the difference quotients in step 3. The accuracy of Method I slightly improves if these nonzero diagonal entries are canceled out, or if $W_{n}$ and $\dot{W}_{n}$ are replaced by their skew-symmetric parts $\left(W_{n}-W_{n}^{T}\right) / 2$ and $\left(\dot{W}_{n}-\dot{W}_{n}^{T}\right) / 2$, respectively.

Remark 3. A starting step can be performed by putting

$$
\eta_{1}=\eta_{0}+h \mathcal{A}_{0} \eta_{0}+h^{2} \mathcal{B}_{0} \eta_{0}+h^{2} \mathcal{C}_{0} \eta_{0}
$$

and integrating from 0 to 1 instead of from -1 to 1 (see [15] for details). For

$$
\begin{aligned}
W_{0}= & \frac{1}{h}\left(Q\left(t_{\frac{1}{2}}\right)-Q\left(t_{-\frac{1}{2}}\right)\right)^{T} Q\left(t_{0}\right), \\
\dot{W}_{0}= & \frac{4}{h^{2}}\left(Q\left(t_{\frac{1}{2}}\right)-2 Q\left(t_{0}\right)+Q\left(t_{-\frac{1}{2}}\right)\right)^{T} Q\left(t_{0}\right) \\
& +\frac{1}{h^{2}}\left(Q\left(t_{\frac{1}{2}}\right)-Q\left(t_{-\frac{1}{2}}\right)\right)^{T}\left(Q\left(t_{\frac{1}{2}}\right)-Q\left(t_{-\frac{1}{2}}\right)\right), \\
\Phi_{1}= & \frac{h}{6}\left(\Lambda\left(t_{0}\right)+4 \Lambda\left(t_{\frac{1}{2}}\right)+\Lambda\left(t_{1}\right)\right), \\
\dot{\Lambda}_{0}= & \frac{1}{h}\left(\Lambda\left(t_{\frac{1}{2}}\right)-\Lambda\left(t_{-\frac{1}{2}}\right)\right),
\end{aligned}
$$

two additional evaluations of $H$ at $t_{\frac{1}{2}}=t_{0}+h / 2$ and $t_{-\frac{1}{2}}=t_{0}-h / 2$ are needed. 
4. Method II. As a reward for the tedious calculations in the previous section, we now obtain yet another numerical scheme, named Method II in what follows, with little additional effort.

4.1. A symmetric Magnus method. Magnus methods originate from the observation of Magnus [17] that the solution of a vector-valued differential equation

$$
\dot{u}(t)=A(t) u(t)
$$

with a time-dependent matrix $A(t)$ can formally be represented as

$$
u(t)=\exp (\Omega(t)) u\left(t_{0}\right),
$$

where $\Omega(t)$ is given by the Magnus series

$$
\begin{aligned}
\Omega(t)= & \int_{t_{0}}^{t} A(\tau) d \tau-\frac{1}{2} \int_{t_{0}}^{t}\left[\int_{t_{0}}^{\tau} A(\sigma) d \sigma, A(\tau)\right] d \tau \\
& +\frac{1}{4} \int_{t_{0}}^{t}\left[\int_{t_{0}}^{\tau}\left[\int_{t_{0}}^{\sigma} A(\mu) d \mu, A(\sigma)\right] d \sigma, A(\tau)\right] d \tau \\
& +\frac{1}{12} \int_{t_{0}}^{t}\left[\int_{t_{0}}^{\tau} A(\sigma) d \sigma,\left[\int_{t_{0}}^{\tau} A(\mu) d \mu, A(\tau)\right]\right] d \tau+\cdots
\end{aligned}
$$

Here, the brackets $[\cdot, \cdot]$ denote the commutator $[X, Y]=X Y-Y X$. Unfortunately, the terms of the Magnus series soon become very complicated, because the number of commutators and integrals grows rapidly, but truncating the series yields an approximation up to any order and can be used for the construction of very precise numerical schemes $[11,12,13,14]$ — provided that it is possible to compute the integrals in $\Omega(t)$ with the required accuracy. This, however, is not a straightforward task in our situation. In the case of the electronic Schrödinger equation (1.1), the matrix $A(t)$ equals

$$
A(t)=-\frac{i}{\varepsilon} H(t)
$$

Consequently, any error caused by quadrature is amplified by the factor $\varepsilon^{-j}$ with $j=1$ for the first integral, $j=2$ for the double integral in the second term, and so on. Due to this unpleasant effect, using a high order quadrature rule would be necessary, but this demands too many costly evaluations of $H(t)$.

In [12], modified Magnus methods for oscillating problems were presented. Here, the differential equation is transformed before applying the Magnus approach. The transformation improves the error behavior of the method but makes computing the integrals of the Magnus series more laborious, since $A(t)$ is replaced by an oscillating matrix. Nevertheless, it is assumed in [12] that these integrals can be approximated by quadrature. This is not feasible in our situation, because many evaluations of $H(t)$ per time step would be unavoidable.

If instead of (1.1) the differential equation (2.3) is considered, the matrix $A(t)$ takes the form

$$
A(t)=\exp \left(\frac{i}{\varepsilon} \Phi(t)\right) W(t) \exp \left(-\frac{i}{\varepsilon} \Phi(t)\right)
$$


Again, $A(t)$ oscillates, and discretizing the corresponding integrals by quadrature would become prohibitively expensive, but now the problem can be solved by means of the expansion technique presented in the previous section. It allows us to compute the integrals sufficiently accurate with only one evaluation.

Since the method is supposed to be time-symmetric, we take the mean of one step forward in time and the inverse of one step backward in time and put

$$
\begin{aligned}
\eta_{n+1} & =\exp \left(\Omega_{n}\right) \eta_{n-1}, \quad \Omega_{n}=\left(\Omega_{n}^{+}+\Omega_{n}^{-}\right) / 2, \\
\Omega_{n}^{ \pm} & =h \int_{-1}^{1} A\left(t_{n}+\theta h\right) d \theta \mp \frac{h^{2}}{2} \int_{-1}^{1}\left[\int_{-1}^{\theta} A\left(t_{n} \pm \sigma h\right) d \sigma, A\left(t_{n} \pm \theta h\right)\right] d \theta, \\
A(t) & =E(\Phi(t)) \bullet W(t) .
\end{aligned}
$$

Now $\Phi\left(t_{n} \pm \theta h\right)$ and $W\left(t_{n} \pm \theta h\right)$ are replaced by the corresponding Taylor expansions as in the previous section. These substitutions lead to terms which are identical or very similar to (3.13), (3.14), and (3.15) and thus can be computed mutatis mutandis. We omit the details and state the algorithm of Method II.

\subsection{The algorithm of Method II.}

1 to 5 . Proceed as in Method I.

6. Compute $\mathcal{A}_{n}$ and $\mathcal{B}_{n}$ as in Method I, and

$$
\begin{aligned}
\mathcal{T}_{6}^{ \pm}\left(t_{n}\right)=E(\mp & \left.h \Lambda\left(t_{n}\right)+\frac{1}{2} h^{2} \dot{\Lambda}_{n}\right) \\
\mathcal{C}_{n}^{ \pm}= \pm \frac{1}{4}( & \mathcal{T}_{4}\left(t_{n}\right) \bullet\left[W_{n}, \pm \mathcal{T}_{5}\left(t_{n}\right)\right] \\
& \left.\quad+\left[E\left(\Phi_{n}\right) \bullet \mathcal{T}_{5}\left(t_{n}\right) \bullet \mathcal{T}_{6}^{ \pm}\left(t_{n}\right), \pm E\left(\Phi_{n}\right) \bullet \mathcal{T}_{1}\left(t_{n}\right) \bullet \mathcal{T}_{5}\left(t_{n}\right)\right]\right) .
\end{aligned}
$$

7. Compute $\Omega_{n}$ and update the quantum vector:

$$
\begin{aligned}
\Omega_{n} & =h \mathcal{A}_{n}+h^{2} \mathcal{B}_{n}+h^{2}\left(\mathcal{C}_{n}^{+}+\mathcal{C}_{n}^{-}\right) / 2, \\
\eta_{n+1} & =\exp \left(\Omega_{n}\right) \eta_{n-1} .
\end{aligned}
$$

8 to 9 . Proceed as in Method I.

Remark 4. Since $W_{n}$ and $\dot{W}_{n}$ are nearly skew-symmetric (cf. Remark 2), $\Omega_{n}$ is nearly skew-Hermitian, and hence Method II almost preserves the norm of the initial value. Exact norm conservation can easily be obtained by replacing $W_{n}$ and $\dot{W}_{n}$ by their corresponding skew-symmetric part, i.e., $\left(W_{n}-W_{n}^{T}\right) / 2$ and $\left(\dot{W}_{n}-\dot{W}_{n}^{T}\right) / 2$, respectively. This slightly improves the accuracy.

Remark 5. Unlike Method I, Method II is not a two-step method but a "shifted" one-step scheme: $\eta_{n}$ is computed from $\eta_{n-2}, \eta_{n+1}$ is computed from $\eta_{n-1}$, and so on. Instead, it seems somewhat more natural to develop a scheme of the type $\eta_{n+1}=$ $\exp \left(\Omega_{n}\right) \eta_{n}$, where the $\eta_{k}$ are approximated successively. This may be done, of course, if $H(t)$ can be evaluated between the time steps, i.e., at $t_{n}+h / 2$, but this is not possible if the Schrödinger equation (1.1) is coupled to some other equation, as is the case, for example, in the surface hopping approach or the QCMD model.

Remark 6. A starting step can be obtained as for Method I or by modifying the Magnus step accordingly. 

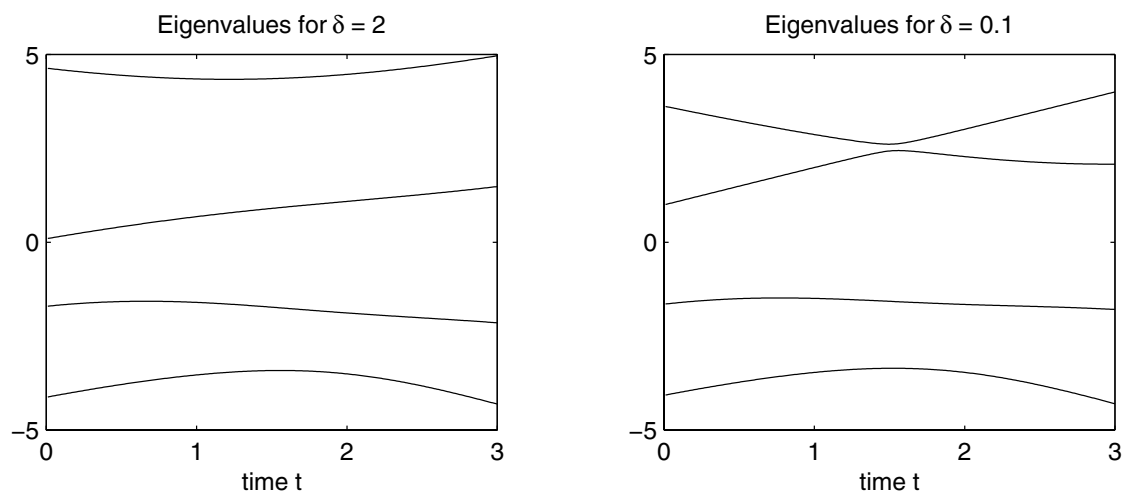

FIG. 5.1. Eigenvalues of $H(t)$ for $\delta=2$ (left-hand side) and $\delta=0.1$ (right-hand side).
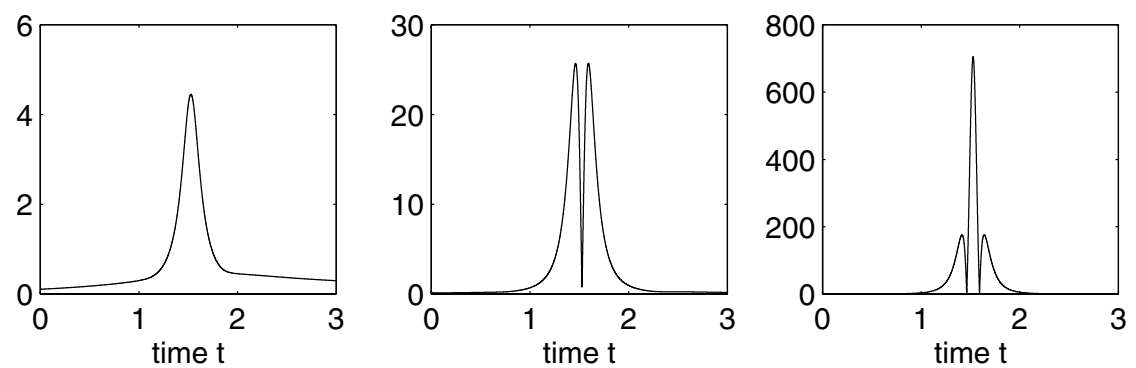

FiG. 5.2. $\|W(t)\|,\|\dot{W}(t)\|$ and $\|\ddot{W}(t)\|$ (from left to right) for $\delta=0.1$.

5. Numerical examples. In this section, the performance of Methods I and II is illustrated by applying them to a test problem which, in spite of its simplicity, reveals characteristic features that appear also in higher-dimensional situations. Let us consider the tridiagonal matrix

$$
H(t)=\left(\begin{array}{cccc}
t+1 & \delta & 0 & 0 \\
\delta & 3-t & 2 & 0 \\
0 & 2 & t-3 & 1 \\
0 & 0 & 1 & h_{44}(t)
\end{array}\right)
$$

with $h_{44}(t)=-4+2 \cos ((2 t-1) \pi / 10)$. The parameter $\delta>0$ exerts a strong influence on how the eigendecomposition of $H(t)$ and the solution of (2.5) behave. Two situations will be distinguished:

- For $\delta=2$, the eigenvalues are well separated at all times $t$ (cf. Figure 5.1, left-hand side), and the norms of the coupling matrix $W(t)$ and its derivatives remain rather small.

- For $\delta=0.1$, the first pair of eigenvalues passes through a so-called avoided crossing (Figure 5.1, right-hand side): The eigenvalues approach each other until $t \approx 1.5$, where they almost intersect, and then separate again. This behavior is accompanied by a sudden increase of coupling between the corresponding components, as is reflected in Figure 5.2 by the high peaks of $\|W(t)\|$ and its derivatives. 

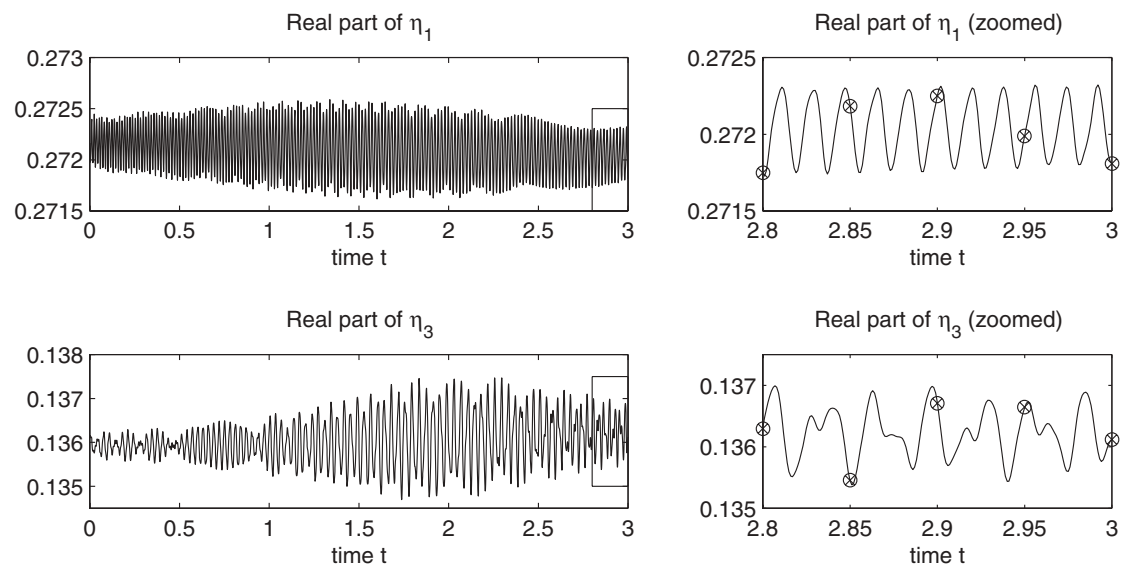

Fig. 5.3. Left-hand side: First and third entries of the exact solution. Right-hand side: Zoom to the areas marked by the boxes. Exact solution (solid lines), Method I (circles) and-almost identical-Method II (crosses) being computed on $[0,3]$ for $\varepsilon=0.01, \delta=2$, and $h=0.05$.

In both cases, solutions of the reformulated equation (2.5) are computed on the interval $[0,3]$ with $\varepsilon=0.01$. For lack of space, only the real part of the first and third entries of $\eta:[0,3] \longrightarrow \mathbb{C}^{4}$ will be displayed wherever the solution is shown.

5.1. First example: $\boldsymbol{\delta}=\mathbf{2}$. This situation is often referred to as the adiabatic case. Figure 5.3 shows two of the real parts of the exact solution (solid lines). As predicted by the quantum-adiabatic theorem (2.4), the range of $\eta$ is only of magnitude $\mathcal{O}(\varepsilon)$. Within this range, $\eta$ oscillates rapidly. On the right-hand side, the region marked by the boxes is enlarged, and the approximations generated by Method I (circles) and Method II (crosses) are added. Both methods work with great precision, even though the step size is five times larger than $\varepsilon$, and within one time step the true solution performs two entire periods of oscillation.

In Figure 5.4, the error is plotted in logarithmic scale versus the step size, and the methods are compared to other numerical schemes (see [15] or [16] for details). All these methods have in common that only one evaluation of $H(t)$ per time step is needed, and that the error is of $\mathcal{O}\left(h^{2}\right)$ as the step size is reduced to $h \ll \varepsilon$. However, as the figure exhibits, the differences in accuracy are immense.

Consider the left-hand side of Figure 5.4. The upper dotted line is the error that results from applying the trapezoidal rule to the original Schrödinger equation $i \varepsilon \dot{\psi}(t)=H(t) \psi(t)$. It demonstrates what happens when an oscillatory problem is tackled in a rather straightforward manner: Only for very small $h \ll \varepsilon$, the error starts to decrease, because the oscillations have to be resolved by tiny step sizes, requiring so many evaluations of $H(t)$ that the computations become prohibitively expensive.

The other two dotted lines (almost identical) were produced by the exponential midpoint rule and again the trapezoidal rule, but now applied to the equation $\dot{\eta}=$ $(E(\Phi) \bullet W) \eta$. Evidently, the reformulation contributes to reduce the error, but the accuracy is still very modest.

The next three solid lines correspond to the integrators proposed in [16], named Method 1, 2, and 3. These are akin to Method I, but certain terms are omitted, saving numerical costs while sacrificing precision. Even Method 1, a very rough 

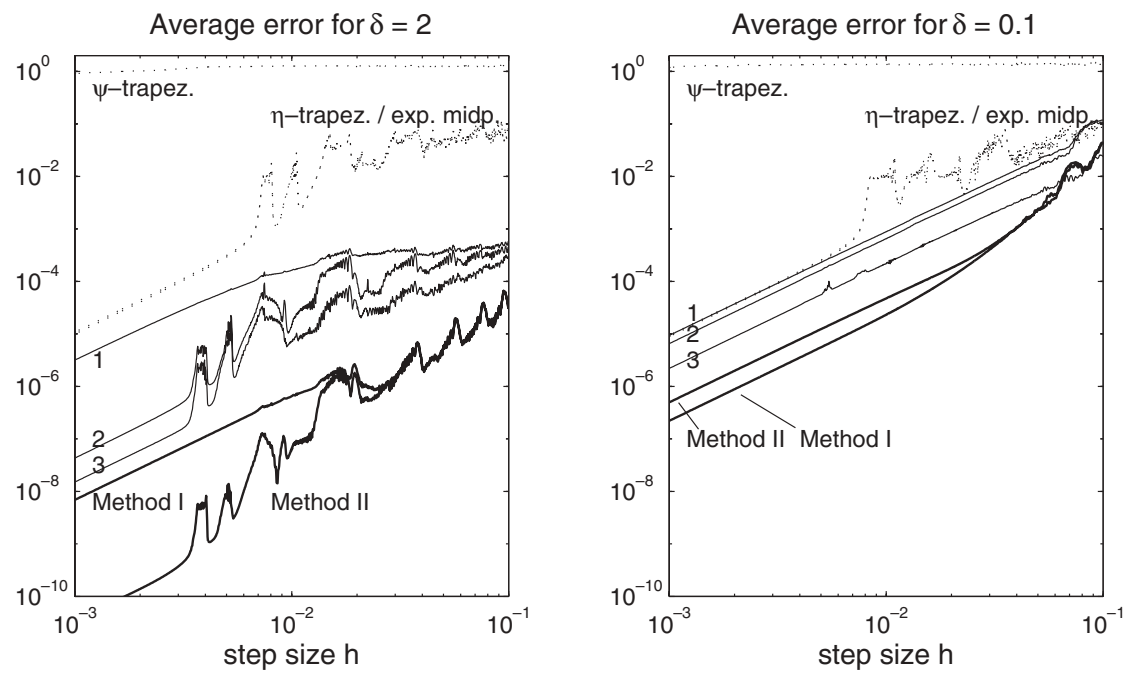

FIG. 5.4. Error of Methods I and II (thick solid lines), compared to other schemes: the trapezoidal rule with respect to $\psi$ and $\eta$ (dotted), the exponential midpoint rule (dotted), and Methods 1,2 , and 3 from [16] (thin solid). Left-hand side: $\delta=2$; right-hand side: $\delta=0.1$.

scheme which was introduced rather for the sake of comparison than for effective computations, performs better than the three traditional solvers.

The best approximations are provided by Methods I and II (thick solid lines). The plot shows that both methods generate similar results for $h>\varepsilon=0.01$, the situation we are interested in. For $h \leq \varepsilon=0.01$, the error of the Magnus-type integrator is significantly smaller.

It should be noticed that in the right half of Figure 5.4, where $h>\varepsilon=0.01$, the accuracy of most of the schemes is subject to resonances for certain values of $h$. As for Methods 2 and 3, the error goes up for certain step sizes, deviating the error curve from a straight line of slope 2 . This phenomenon was analyzed, explained, and controlled in $[15,16]$. Methods I and II are robust to such resonance effects. In fact, their error is clearly of $\mathcal{O}\left(h^{2}\right)$.

5.2. Second example: $\boldsymbol{\delta}=\mathbf{0 . 1}$. Figure 5.5 shows what happens if $\delta$ is reduced to 0.1 and the first two eigenvalues $\lambda_{1}(t)$ and $\lambda_{2}(t)$ pass through an avoided crossing. Around $t \approx 1.5$, where the gap between these eigenvalues is tiny, the quantumadiabatic theorem (2.4) ceases to be valid. Thus, the entries $\eta_{1}(t)$ and $\eta_{2}(t)$ may leave their range of $\mathcal{O}(\varepsilon)$ and "jump" to completely new levels. As an example, the real part of $\eta_{1}(t)$ is shown in Figure 5.5 (upper two plots). The nonadiabatic transition is visible on a scale of $\mathcal{O}(1)$, whereas outside the avoided crossing region, the solution appears almost as a straight line. However, a strong zoom reveals small oscillations of $\eta(t)$ within an $\mathcal{O}(\varepsilon)$ range as in the adiabatic case; cf. the upper right picture in Figure 5.5.

How do the numerical methods cope with this completely new behavior of the solution? Before the nonadiabatic transitions, the situation is similar to the adiabatic case, and Methods I and II give very exact results. Within the avoided crossing, accuracy is reduced due to the nonsmooth behavior of the first two entries. The error arises from three sources. First, from the fact that both methods approximate the coupling matrix $W(t)$ only linearly, although $W(t)$ changes rapidly during the avoided 

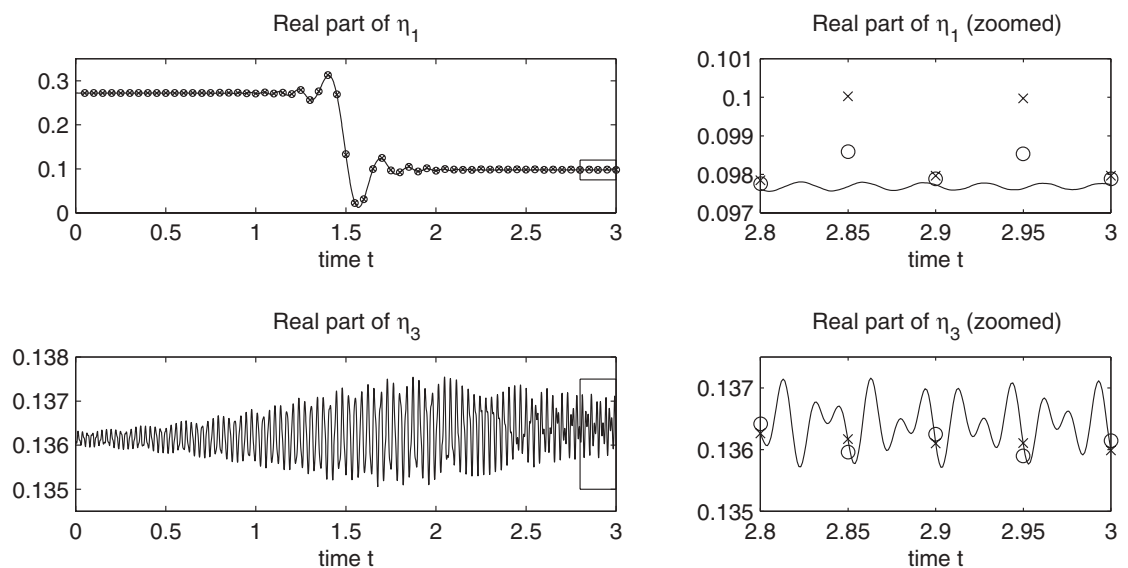

FIG. 5.5. Left-hand side: First and third entries of the exact solution, upper left with the approximations of the methods included. Right-hand side: Zoom to the areas marked by the boxes. Exact solution (solid lines), Method I (circles), and Method II (crosses) being computed on [0,3] for $\varepsilon=0.01, \delta=0.1$, and $h=0.05$.

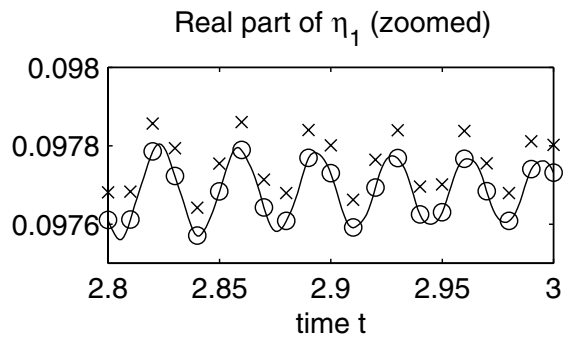

Real part of $\eta_{3}$ (zoomed)

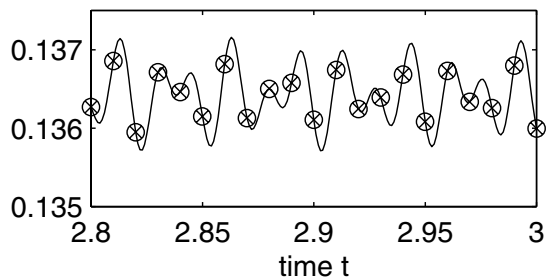

FIG. 5.6. Same situation as in Figure 5.5, but with the step size reduced to $h=\varepsilon=0.01$.

crossing (cf. Figure 5.2). Second, from the matrix $D^{-}\left(\Lambda\left(t_{n}\right)\right)$ containing, by definition, $\pm 1 /\left(\lambda_{1}-\lambda_{2}\right)$ as two of its entries, which are large during the avoided crossings and multiply errors. Third, from the difference quotients in steps 3 and 4 giving less precise approximations now that the corresponding functions $W, \dot{W}$, and $\dot{\Lambda}$ are not smooth.

Nevertheless, both integrators depict the "jump behavior" on the large scale correctly even for big step sizes; cf. the left upper plot of Figure 5.5. Only the tiny oscillations on the small scale after the avoided crossing are not reproduced as well as before, cf. the upper right picture in Figure 5.5. However, if the $h$ is decreased to $h=\delta^{2}=\varepsilon=0.01$, Method I approximates even these oscillations very well, as is shown in Figure 5.6. 
This suggests using an adaptive version of Method I and II and reducing the step size automatically near the avoided crossing. Adaptive versions of Methods 13 were presented in [16] and can be devised for the new integrators as well, but in several numerical tests, neither Method I nor Method II produced a better result with an adaptive step size than with a fixed one. The reason is that some error terms arising from the integral expansions (cf. section 3.2) almost vanish due to the symmetric structure when equidistant approximations are computed but can increase when symmetry is traded for adaptivity.

The entries $\eta_{3}(t)$ and $\eta_{4}(t)$ are not affected by the avoided crossing, because their eigenvalues remain separated from the rest of the spectrum. Hence, they behave as in the first example and are computed with good precision by both methods. This is demonstrated in the lower two pictures of Figure 5.5, where the real part of $\eta_{3}(t)$ is plotted.

The right-hand side of Figure 5.4 again shows a comparison between Methods I and II and the other numerical schemes as described above. Most of the graphs are much smoother than in the first example, because now the main error does not originate from the small scale oscillations and resonances, but from the nonadiabatic transitions on the large scale. Again, Methods I and II clearly achieve the best results, but in contrast to the first example, it is now the former which performs better than the latter with respect to the average error.

6. Error analysis. According to Figure 5.4, the new integrators seem to be of second order even for $h>\varepsilon$. This observation is confirmed mathematically in this section.

\subsection{Error of Method I.}

THEOREM 6.1. Suppose that $\varepsilon>0$, and choose the step size $h>0$ such that $\varepsilon<h<\sqrt{\varepsilon}$ holds. Let $\psi_{n}$ and $\eta_{n}$ denote the approximations given by Method I. Then, under the conditions of subsection 2.1 , the errors at $t_{n}=t_{0}+n h \in\left[t_{0}, t_{\text {end }}\right]$ are bounded by

$$
\begin{aligned}
\left\|\eta\left(t_{n}\right)-\eta_{n}\right\| & \leq C_{1} h^{2}, \\
\left\|\psi\left(t_{n}\right)-\psi_{n}\right\| & \leq C_{2} h^{2} .
\end{aligned}
$$

The constants $C_{1}$ and $C_{2}$ depend on bounds of the first three derivatives of $Q$ and $\Lambda$, on $\delta_{\min }$ and $t_{\text {end }}-t_{0}$ but are independent of $\varepsilon$ and $h$.

Proof of Theorem 6.1. Let $n \in \mathbb{N}$ be fixed. The main task is to prove that the local error is bounded by $\mathcal{O}\left(h^{3}\right)$, i.e.,

$$
\begin{aligned}
& \| \eta\left(t_{n+1}\right)-\left(\eta\left(t_{n-1}\right)+h \mathcal{A}_{n} \eta\left(t_{n}\right)\right. \\
& \left.\quad+h^{2} \mathcal{B}_{n} \eta\left(t_{n}\right)+h^{2} \mathcal{C}_{n} \eta\left(t_{n}\right)\right) \| \leq C h^{3} .
\end{aligned}
$$

The rest of the proof follows from a discrete version of Gronwall's lemma, and the estimate for $\psi_{n}$ is a consequence of the transformation (2.2) with $\Phi_{n}$ substituting $\Phi\left(t_{n}\right)$. For simplicity, these abbreviations will be used:

- Let $\widehat{\alpha}_{n}, \widehat{\beta}_{n}$, and $\widehat{\gamma}_{n}$ be defined as in (3.4), (3.5), and (3.6), respectively, but with $W\left(t_{n}\right)$ replaced by $W_{n}$, and $\dot{W}\left(t_{n}\right)$ replaced by $\dot{W}_{n}$.

- Let $\check{\alpha}_{n}, \breve{\beta}_{n}$, and $\check{\gamma}_{n}$ be defined as in (3.13), (3.14), and (3.15), respectively, but with $\Phi\left(t_{n}\right)$ instead of $\Phi_{n}$, and $\dot{\Lambda}\left(t_{n}\right)$ instead of $\dot{\Lambda}_{n}$. 
The local error can be split into the four parts

$$
\begin{aligned}
\eta\left(t_{n+1}\right) & -\left(\eta\left(t_{n-1}\right)+h \mathcal{A}_{n} \eta\left(t_{n}\right)+h^{2} \mathcal{B}_{n} \eta\left(t_{n}\right)+h^{2} \mathcal{C}_{n} \eta\left(t_{n}\right)\right) \\
= & \eta\left(t_{n+1}\right)-\left(\eta\left(t_{n-1}\right)+h \widehat{\alpha}_{n} \eta\left(t_{n}\right)+h^{2} \widehat{\beta}_{n} \eta\left(t_{n}\right)+h^{2} \widehat{\gamma}_{n} \eta\left(t_{n}\right)\right) \\
& +\left(h \widehat{\alpha}_{n}+h^{2} \widehat{\beta}_{n}+h^{2} \widehat{\gamma}_{n}\right) \eta\left(t_{n}\right)-\left(h \check{\alpha}_{n}+h^{2} \check{\beta}_{n}+h^{2} \check{\gamma}_{n}\right) \eta\left(t_{n}\right) \\
& +\left(h \check{\alpha}_{n}+h^{2} \check{\beta}_{n}+h^{2} \check{\gamma}_{n}\right) \eta\left(t_{n}\right)-\left(h \widetilde{\alpha}_{n}+h^{2} \widetilde{\beta}_{n}+h^{2} \widetilde{\gamma}_{n}\right) \eta\left(t_{n}\right) \\
& +\left(h \widetilde{\alpha}_{n}+h^{2} \widetilde{\beta}_{n}+h^{2} \widetilde{\gamma}_{n}\right) \eta\left(t_{n}\right)-\left(h \mathcal{A}_{n}+h^{2} \mathcal{B}_{n}+h^{2} \mathcal{C}_{n}\right) \eta\left(t_{n}\right)
\end{aligned}
$$

with the following interpretation: (6.2) is the error that originates from replacing the intermediate values of $W$ via (3.3), (3.7), and (3.8). Substituting the Taylor expansion (3.10) for $\Phi\left(t_{n}+\theta h\right)$ causes the error (6.3). Computing $\Phi\left(t_{n}\right)$ by the Simpson rule (3.12) and $\dot{\Lambda}\left(t_{n}\right)$ by the difference quotient (3.11) yields (6.4). The difference (6.5) is introduced by the integral expansions from section 3.2.

For the rest of this paper, $C$ denotes constants which are not distinguished, and instead of

$$
\sup _{t}\|W(t)\|, \quad \sup _{t}\|\ddot{\Lambda}(t)\|, \quad \sup _{t}\left\|\frac{\partial^{3}}{\partial t^{3}} Q(t)\right\|,
$$

with the suprema taken over all $t \in\left[t_{0}, t_{\text {end }}\right]$, we use the short forms

$$
\|W\|, \quad\|\ddot{\Lambda}\|, \quad\left\|\partial^{3} Q\right\|, \quad \text { etc. }
$$

6.1.1. Estimating (6.2). Standard arguments show that the errors of the approximations (3.7) and (3.8) are

$$
\begin{gathered}
\left\|W\left(t_{n}\right)-W_{n}\right\| \leq C h^{2}\left\|\partial^{3} Q\right\|, \\
\left\|\dot{W}\left(t_{n}\right)-\dot{W}_{n}\right\| \leq C h\left\|\partial^{3} Q\right\|,
\end{gathered}
$$

which yields

$$
\begin{aligned}
\left\|W\left(t_{n}+\theta h\right)-W_{n}-\theta h \dot{W}_{n}\right\| & \leq C h^{2}\left(\left\|\partial^{3} Q\right\|+\|\ddot{W}\|\right), \\
\left\|W\left(t_{n}+\theta h\right)-W_{n}\right\| & \leq C h\|\dot{W}\|+\mathcal{O}\left(h^{2}\right) .
\end{aligned}
$$

Furthermore, the estimate $\left\|\eta\left(t_{n}+\sigma h\right)-\eta\left(t_{n}\right)\right\| \leq h \sigma\|W\|$ is available from

$$
\eta\left(t_{n}+\sigma h\right)-\eta\left(t_{n}\right)=h \int_{0}^{\sigma}\left(E\left(\Phi\left(t_{n}+\xi h\right)\right) \bullet W\left(t_{n}+\xi h\right)\right) \eta\left(t_{n}+\xi h\right) d \xi .
$$

With these inequalities, it follows that (6.2) is bounded by

$$
C h^{3}\left(\left\|\partial^{3} Q\right\|+\|\ddot{W}\|+\|\dot{W}\|\|W\|+\|W\|^{3}\right)+\mathcal{O}\left(h^{4}\right) .
$$

6.1.2. Estimating (6.3). Let the remainder of the Taylor expansion (3.10) be denoted by $\theta^{3} h^{3} R(t, \theta h)$, i.e.,

$$
\begin{aligned}
\Phi(t+\theta h) & =\Phi(t)+\theta h \Lambda(t)+\frac{1}{2} \theta^{2} h^{2} \dot{\Lambda}(t)+\theta^{3} h^{3} R(t, \theta h), \\
\|R(t, \theta h)\| & \leq C\|\ddot{\Lambda}\| .
\end{aligned}
$$


Moreover, a matrix-valued function is defined by

$$
F(t, x)=\frac{\varepsilon}{i x^{3}}\left(E\left(x^{3} R(t, x)\right)-E(0)\right)=D(R(t, x)) \bullet \int_{0}^{1} E\left(\xi x^{3} R(t, x)\right) d \xi
$$

for $x \neq 0$ and by the corresponding limit for $x=0 . F$ is continuously differentiable with respect to $t$ and bounded by

$$
\|F(t, x)\|=C\|R(t, x)\| \leq C\|\ddot{\Lambda}\| .
$$

With this notation, we can write

$$
\begin{aligned}
& \left(h \widehat{\alpha}_{n}+h^{2} \widehat{\beta}_{n}\right)-\left(h \check{\alpha}_{n}+h^{2} \check{\beta}_{n}\right) \\
& =h E\left(\Phi\left(t_{n}\right)\right) \bullet \int_{-1}^{1} E\left(\theta h \Lambda\left(t_{n}\right)+\frac{1}{2} \theta^{2} h^{2} \dot{\Lambda}\left(t_{n}\right)\right) \bullet \frac{i \theta^{3} h^{3}}{\varepsilon} F\left(t_{n}, \theta h\right) d \theta \bullet W_{n}
\end{aligned}
$$

and integrate by parts:

$$
\begin{aligned}
& \int_{-1}^{1} E\left(\theta h \Lambda\left(t_{n}\right)+\frac{1}{2} \theta^{2} h^{2} \dot{\Lambda}\left(t_{n}\right)\right) \bullet \frac{i \theta^{3} h^{3}}{\varepsilon} F\left(t_{n}, \theta h\right) d \theta \\
& =\left.\int_{-1}^{\theta} E\left(\sigma h \Lambda\left(t_{n}\right)\right) d \sigma \bullet E\left(\frac{1}{2} \theta^{2} h^{2} \dot{\Lambda}\left(t_{n}\right)\right) \bullet \frac{i \theta^{3} h^{3}}{\varepsilon} F\left(t_{n}, \theta h\right)\right|_{\theta=-1} ^{\theta=1} \\
& \quad-\int_{-1}^{1} \int_{-1}^{\theta} E\left(\sigma h \Lambda\left(t_{n}\right)\right) d \sigma \bullet \frac{d}{d \theta}\left(E\left(\frac{1}{2} \theta^{2} h^{2} \dot{\Lambda}\left(t_{n}\right)\right) \bullet \frac{i \theta^{3} h^{3}}{\varepsilon} F\left(t_{n}, \theta h\right)\right) d \theta .
\end{aligned}
$$

With

$$
\int_{-1}^{\theta} E\left(\sigma h \Lambda\left(t_{n}\right)\right) d \sigma=\frac{\varepsilon}{i h} D^{-}\left(\Lambda\left(t_{n}\right)\right) \bullet\left[E\left(\theta h \Lambda\left(t_{n}\right)\right)-E\left(-h \Lambda\left(t_{n}\right)\right)\right]
$$

and

$$
\begin{aligned}
& \frac{d}{d \theta}\left(E\left(\frac{1}{2} \theta^{2} h^{2} \dot{\Lambda}\left(t_{n}\right)\right) \bullet \frac{i \theta^{3} h^{3}}{\varepsilon} F\left(t_{n}, \theta h\right)\right) \\
& =\frac{i}{\varepsilon} \theta h^{2} D\left(\dot{\Lambda}\left(t_{n}\right)\right) \bullet E\left(\frac{1}{2} \theta^{2} h^{2} \dot{\Lambda}\left(t_{n}\right)\right) \bullet \frac{i \theta^{3} h^{3}}{\varepsilon} F\left(t_{n}, \theta h\right) \\
& \quad+E\left(\frac{1}{2} \theta^{2} h^{2} \dot{\Lambda}\left(t_{n}\right)\right) \bullet \frac{i h^{3}}{\varepsilon} \frac{d}{d \theta}\left(\theta^{3} F\left(t_{n}, \theta h\right)\right),
\end{aligned}
$$

this enables the estimate

$$
\left\|\left(h \widehat{\alpha}_{n}+h^{2} \widehat{\beta}_{n}\right) \eta\left(t_{n}\right)-\left(h \check{\alpha}_{n}+h^{2} \check{\beta}_{n}\right) \eta\left(t_{n}\right)\right\| \leq C \frac{h^{3}}{\delta_{\min }}\|\ddot{\Lambda}\|\|W\|+\mathcal{O}\left(h^{4}\right) .
$$

Here we have used that, by assumption, $\frac{h^{2}}{\varepsilon} \leq 1$, and that

$$
\begin{aligned}
\left\|D\left(\dot{\Lambda}\left(t_{n}\right)\right)\right\| & \leq C, & \left\|F\left(t_{n}, \theta h\right)\right\| & \leq C\|\ddot{\Lambda}\|, \\
\left\|D^{-}\left(\Lambda\left(t_{n}\right)\right)\right\| & \leq C / \delta_{\min }, & \left\|\frac{d}{d \theta}\left(\theta^{3} F\left(t_{n}, \theta h\right)\right)\right\| & \leq 3 \theta^{2}\left\|F\left(t_{n}, \theta h\right)\right\|+\mathcal{O}(h) .
\end{aligned}
$$


The difference $\widehat{\gamma}_{n}-\check{\gamma}_{n}$ can be rewritten as

$$
\begin{aligned}
&\left(\int_{-1}^{1} E\left(\Phi\left(t_{n}\right)+\theta h \Lambda\left(t_{n}\right)+\frac{1}{2} \theta^{2} h^{2} \dot{\Lambda}\left(t_{n}\right)\right)\right.\left.\bullet \frac{i}{\varepsilon} \theta^{3} h^{3} F\left(t_{n}, \theta h\right) \bullet W_{n}\right) \\
& \times \int_{0}^{\theta}\left(E\left(\Phi\left(t_{n}+\sigma h\right)\right) \bullet W_{n}\right) d \sigma d \theta \\
&+\left(\int_{-1}^{1} E\left(\Phi\left(t_{n}\right)+\theta h \Lambda\left(t_{n}\right)+\frac{1}{2} \theta^{2} h^{2} \dot{\Lambda}\left(t_{n}\right)\right)\right.\left.\bullet W_{n}\right) \\
& \times\left(\int_{0}^{\theta} E\left(\Phi\left(t_{n}\right)+\sigma h \Lambda\left(t_{n}\right)+\frac{1}{2} \sigma^{2} h^{2} \dot{\Lambda}\left(t_{n}\right)\right) \bullet \frac{i}{\varepsilon} \sigma^{3} h^{3} F\left(t_{n}, \sigma h\right) \bullet W_{n}\right) d \sigma d \theta,
\end{aligned}
$$

which shows that

$$
\left\|\widehat{\gamma}_{n}-\check{\gamma}_{n}\right\| \leq C h\|\ddot{\Lambda}\|\|W\|^{2}+\mathcal{O}\left(h^{2}\right),
$$

and hence that (6.3) is bounded by

$$
C h^{3}\|\ddot{\Lambda}\|\left(\|W\| / \delta_{\min }+\|W\|^{2}\right)+\mathcal{O}\left(h^{4}\right) .
$$

6.1.3. Estimating (6.4). The errors of the Simpson rule (3.12) and of the difference quotient (3.11) are

$$
\begin{array}{r}
\left\|\Phi\left(t_{n}\right)-\Phi_{n}\right\| \leq C h^{4}\left\|\partial^{3} \Lambda\right\|, \\
\left\|\dot{\Lambda}(t)-\dot{\Lambda}_{n}\right\| \leq C h^{2}\left\|\partial^{3} \Lambda\right\| .
\end{array}
$$

With $\frac{h^{2}}{\varepsilon} \leq 1$, one concludes that

$$
\left\|\check{\alpha}_{n}-\widetilde{\alpha}_{n}\right\| \leq C h^{2}\left\|\partial^{3} \Lambda\right\|\|W\|,
$$

and since $\left\|\check{\beta}_{n}-\widetilde{\beta}_{n}\right\|$ and $\left\|\check{\gamma}_{n}-\widetilde{\gamma}_{n}\right\|$ produce only higher order errors, this yields the bound $C h^{3}\left\|\partial^{3} \Lambda\right\|\|W\|+\mathcal{O}\left(h^{4}\right)$ for (6.4).

6.1.4. Estimating (6.5). An inspection of (3.16) and straightforward calculations reveal that

$$
\begin{aligned}
& \int_{-1}^{1} \theta E\left(\theta h \Lambda\left(t_{n}\right)+\frac{1}{2} \theta^{2} h^{2} \dot{\Lambda}_{n}\right) d \theta-\mathcal{T}_{3}\left(t_{n}\right) \\
& =-\int_{-1}^{1} E\left(\theta h \Lambda\left(t_{n}\right)+\frac{1}{2} \theta^{2} h^{2} \dot{\Lambda}_{n}\right) \\
& \quad \bullet\left(\theta \frac{\varepsilon}{i h} D^{-}\left(\Lambda\left(t_{n}\right)\right)-\left(\frac{\varepsilon}{i h} D^{-}\left(\Lambda\left(t_{n}\right)\right)\right)^{\bullet 2}\right) \bullet \theta \frac{i h^{2}}{\varepsilon} D\left(\dot{\Lambda}_{n}\right) d \theta \\
& =-h D^{-}\left(\Lambda\left(t_{n}\right)\right) \bullet D\left(\dot{\Lambda}_{n}\right) \\
& \quad \bullet \int_{-1}^{1}\left(\theta E\left(\theta h \Lambda\left(t_{n}\right)\right)+E\left(-h \Lambda\left(t_{n}\right)\right)-\int_{-1}^{\theta} E\left(\sigma h \Lambda\left(t_{n}\right)\right) d \sigma\right) \bullet \theta E\left(\frac{1}{2} \theta^{2} h^{2} \dot{\Lambda}_{n}\right) d \theta,
\end{aligned}
$$

and consequently

$$
\left\|\int_{-1}^{1} \theta E\left(\theta h \Lambda\left(t_{n}\right)+\frac{1}{2} \theta^{2} h^{2} \dot{\Lambda}_{n}\right) d \theta-\mathcal{T}_{3}\left(t_{n}\right)\right\| \leq C \frac{h}{\delta_{\min }} .
$$


From (3.21), it follows that

$$
\left\|\int_{-1}^{1} E\left(\theta h \Lambda\left(t_{n}\right)+\frac{1}{2} \theta^{2} h^{2} \dot{\Lambda}_{n}\right) d \theta-\frac{\varepsilon}{i h} D^{-}\left(\Lambda\left(t_{n}\right)\right) \bullet \mathcal{T}_{1}\left(t_{n}\right)\right\| \leq C \frac{h}{\delta_{\min }},
$$

or, using (6.7), that

$$
\begin{aligned}
& \| \int_{-1}^{1} E\left(\theta h \Lambda\left(t_{n}\right)+\frac{1}{2} \theta^{2} h^{2} \dot{\Lambda}_{n}\right) d \theta \\
& \quad-\left(\frac{\varepsilon}{i h} D^{-}\left(\Lambda\left(t_{n}\right)\right) \bullet \mathcal{T}_{1}\left(t_{n}\right)-h D^{-}\left(\Lambda\left(t_{n}\right)\right) \bullet D\left(\dot{\Lambda}_{n}\right) \bullet \mathcal{T}_{3}\left(t_{n}\right)\right) \| \leq C\left(\frac{h}{\delta_{\min }}\right)^{2} .
\end{aligned}
$$

Inserting the estimates (6.7) and (6.8) now leads to

$$
\left\|\left(h \widetilde{\alpha}_{n}+h^{2} \widetilde{\beta}_{n}\right) \eta\left(t_{n}\right)-\left(h \mathcal{A}_{n}+h^{2} \mathcal{B}_{n}\right) \eta\left(t_{n}\right)\right\| \leq C h^{3}\left(\frac{1}{\delta_{\min }^{2}}\|W\|+\frac{1}{\delta_{\min }}\|\dot{W}\|\right),
$$

and, analogously, it can be shown that

$$
\left\|h^{2} \widetilde{\gamma}_{n} \eta\left(t_{n}\right)-h^{2} \mathcal{C}_{n} \eta\left(t_{n}\right)\right\| \leq C h^{3}\|W\|^{2}\left(\frac{1}{\delta_{\min }^{2}}+\frac{1}{\delta_{\min }}\right) .
$$

This completes the proof of (6.1). By similar calculations, it can be verified that the error of the starting step is $\mathcal{O}\left(h^{2}\right)$.

\subsection{Error of Method II.}

THEOREM 6.2. Under the assumptions of Theorem 6.1, the errors of Method II are bounded by

$$
\begin{aligned}
\left\|\eta\left(t_{n}\right)-\eta_{n}\right\| & \leq C_{1} h^{2}, \\
\left\|\psi\left(t_{n}\right)-\psi_{n}\right\| & \leq C_{2} h^{2}
\end{aligned}
$$

with constants $C_{1}$ and $C_{2}$ depending on the same terms as in Theorem 6.1.

Proof. Classical error analysis states that truncating the Magnus series after the second term yields a fourth order scheme [13]. This does not hold in the case of an oscillating matrix and a large step size, but since all truncated terms contain at least three integrals and since the matrix $E(\Phi) \bullet W$ is uniformly bounded with respect to $\varepsilon$, the local truncation error is of order $\mathcal{O}\left(h^{3}\right)$. The errors induced by the use of Taylor expansions, difference quotients, and approximate evaluation of the integrals can be estimated as was done in the proof of Theorem 6.1.

Acknowledgments. The author thanks Christian Lubich for valuable advice on the subject of this paper and a thorough reading of the manuscript; Arieh Iserles and Jitse Niesen for various discussions on Magnus methods; and the referees for helpful comments.

\section{REFERENCES}

[1] S. Blanes And P. C. Moan, Splitting methods for the time-dependent Schrödinger equation, Phys. Lett. A, 265 (2000), pp. 35-42.

[2] M. Born And V. Fock, Beweis des Adiabatensatzes, Z. Phys., 51 (1928), pp. 165-180. 
[3] F. A. Bornemann, P. Nettesheim, and Ch. SchÜtte, Quantum-classical molecular dynamics as an approximation to full quantum dynamics, J. Chem. Phys., 105 (1996), pp. 1074-1083.

[4] F. A. Bornemann And Ch. Schütte, On the singular limit of the quantum-classical molecular dynamics model, SIAM J. Appl. Math., 59 (1999), pp. 1208-1224.

[5] P. Deuflhard, J. Hermans, B. Leimkuhler, A. E. Mark, S. Reich, and R. D. Skeel, EDs., Computational Molecular Dynamics: Challenges, Methods, Ideas, Lecture Notes in Comput. Sci. Engrg. 4, Springer-Verlag, Berlin, 1999.

[6] N. L. Doltsinis, Nonadiabatic Dynamics: Mean-Field and Surface Hopping, in Quantum Simulations of Complex Many-Body Systems: From Theory to Algorithms, J. Grotendorst, D. Marx, and A. Muramatsu, eds., John von Neumann Institute for Computing, Forschungszentrum Jülich, Jülich, 2002, pp. 377-397.

[7] N. L. Doltsinis AND D. MARx, First principles molecular dynamics involving excited states and nonadiabatic transitions, J. Theor. Comp. Chem., 1 (2002), pp. 319-349.

[8] G. A. Hagedorn, Proof of the Landau-Zener formula in an adiabatic limit with small eigenvalue gaps, Comm. Math. Phys., 136 (1991), pp. 433-449.

[9] M. HochBruck AND CH. Lubich, A bunch of time integrators for quantum/classical molecular dynamics, in Computational Molecular Dynamics: Challenges, Methods, Ideas, Lecture Notes in Comput. Sci. Engrg. 4, Springer-Verlag, Berlin, 1999, pp. 421-432.

[10] M. Hochbruck AND CH. LuBich, Exponential integrators for quantum-classical molecular dynamics, BIT, 39 (1999), pp. 620-645.

[11] M. Hochbruck And Ch. Lubich, On Magnus integrators for time-dependent Schrödinger equations, SIAM J. Numer. Anal., 41 (2003), pp. 945-963.

[12] A. ISERLES, On the global error of discretization methods for highly-oscillatory ordinary differential equations, BIT, 42 (2002), pp. 561-599.

[13] A. Iserles, H. Z. Munthe-KaAs, S. P. Nørsett, And A. Zanna, Lie-group methods, in Acta Numer. 9, Cambridge University Press, Cambridge, UK, 2000, pp. 215-365.

[14] A. Iserles AND S. P. NøRSETt, On the solution of linear differential equations in Lie groups, R. Soc. Lond. Philos. Trans. Ser. A Math. Phys. Eng. Sci., 357 (1999), pp. 983-1020.

[15] T. Jahnke, Numerische Verfahren für fast adiabatische Quantendynamik, Ph.D. thesis, Universität Tübingen, Germany, 2003.

[16] T. Jahnke AND CH. LuBich, Numerical integrators for quantum dynamics close to the adiabatic limit, Numer. Math., 94 (2003), pp. 289-314.

[17] W. Magnus, On the exponential solution of differential equations for a linear operator, Comm. Pure Appl. Math., 7 (1954), pp. 649-673.

[18] D. Marx AND J. HutTer, Ab initio molecular dynamics: Theory and implementation, in Modern Methods and Algorithms of Quantum Chemistry, J. Grotendorst, ed., John von Neumann Institute for Computing, Forschungszentrum Jülich, Jülich, 2000, pp. 329-477.

[19] P. Nettesheim And Ch. Schütte, Numerical integrators for quantum-classical molecular $d y$ namics, in Computational Molecular Dynamics: Challenges, Methods, Ideas, Lecture Notes in Comput. Sci. Engrg. 4, Springer-Verlag, Berlin, 1999, pp. 396-411.

[20] P. Nettesheim, Mixed Quantum-Classical Dynamics: A Unified Approach to Mathematical Modelling and Numerical Simulation, Ph.D. thesis, Freie Universität Berlin, 2000.

[21] J. C. TUlly Und R. K. Preston, Trajectory surface hopping approach to nonadiabatic molecular collisions: The reaction of $\mathrm{H}^{+}$with $\mathrm{D}_{2}$, J. Chem. Phys., 55 (1971), pp. 562-572.

[22] C. Zener, Nonadiabatic crossing of energy levels, Proc. Roy. Soc. London Ser. A, 137 (1932), pp. 696-702. 